\title{
MODAL ANALYSIS AND MODELING OF A FRICTIONLESS ELECTROSTATIC ROTARY STEPPER MICROMOTOR
}

\author{
M. Stranczl ${ }^{1}$, E. Sarajlic ${ }^{2}$, G.J.M. Krijnen ${ }^{3}$, H. Fujita ${ }^{4}$, M.A.M. Gijs ${ }^{1}$, and C. Yamahata ${ }^{1}$ \\ ${ }^{1}$ Laboratory of Microsystems, Ecole Polytechnique Fédérale de Lausanne (EPFL), SWITZERLAND, \\ ${ }^{2}$ SmartTip B.V., Enschede, THE NETHERLANDS, \\ ${ }^{3} \mathrm{MESA}+$, University of Twente, Enschede, THE NETHERLANDS, \\ ${ }^{4}$ CIRMM, Institute of Industrial Science, The University of Tokyo, Tokyo, JAPAN
}

\begin{abstract}
We present the design, modeling and characterization of a 3-phase electrostatic rotary stepper micromotor. The proposed motor is a monolithic device fabricated using silicon-on-insulator (SOI) technology. The rotor is suspended with a frictionless flexural pivot bearing and reaches an unprecedented rotational range of $30^{\circ}\left(+/-15^{\circ}\right)$ at $65 \mathrm{~V}$. We have established a mechanical model of the deformation structure and performed finite element analysis (FEA) simulations of the dynamic properties. These studies are consistent with the extensive experimental characterization performed in the quasi-static, transient, and dynamic regimes.
\end{abstract}

\section{INTRODUCTION}

We have recently developed a piggyback stepper micromotor aimed at skew angle compensation in hard disk drives [1]. The mechanism of this frictionless motor is based on the monolithic "butterfly" pivot devised by Henein et al. $[2,3]$. With a reachable rotational range of $+/-15^{\circ}$, this type of microactuator can be advantageously used in many MEMS applications. In this perspective, we have developed a mechanical model to optimize the static and dynamic responses of the motor. With this model, we have designed a new flexural bearing with a lower inertia and a higher outof-plane stiffness. The improved design results in a very large displacement range, and an opening in the stator enables the inclusion of an arm required for microrobotic applications. In Figure 1, the arm is represented by a needle that indicates the angular displacement.

\section{WORKING PRINCIPLE}

The left schematic in Figure 1 shows the working principle and the main elements of the SOI electrostatic stepper micromotor. The motor consists of a 3-phase stator and a grounded rotor which is suspended by a flexural mechanism [2]. This frictionless bearing enables a rotational degree of freedom with a certain torsional stiffness. The stator electrodes are grouped into three pairs that are symmetrically located around the rotor (see the photograph in Figure 1). Each phase can be activated independently (voltages $U_{1}, U_{2}$ and $U_{3}$ ). In the initial position, the electrodes of the first phase are perfectly aligned with the opposite electrodes on the rotor. The stator electrodes of the two other phases have a misalignment which is equal to $1 / 3$ of the pitch of the rotor electrodes.

\section{MODELING}

\section{Flexural pivot stiffness}

Using material mechanics theory, with the assumption that the material deformation is purely elastic and isotropic, we find that the stiffness of the 4-beam flexural pivot shown in Figure 1(right) is given by:

$$
K_{\theta}=E I \frac{a^{2}+a l+4\left(l^{2}+3 l p+3 p^{2}\right)}{(l-a)\left(a^{2}+a l+l^{2}\right)}, I=\frac{h b^{3}}{12},
$$

where $E$ is the Young's modulus of the beam material, $h$ is the vertical height, $b$ is the width of the flexure hinges, $a$ is the length of the central reinforced structure, $l$ is the total length of the beam elements, and $p$ is their distance to the virtual center of rotation.
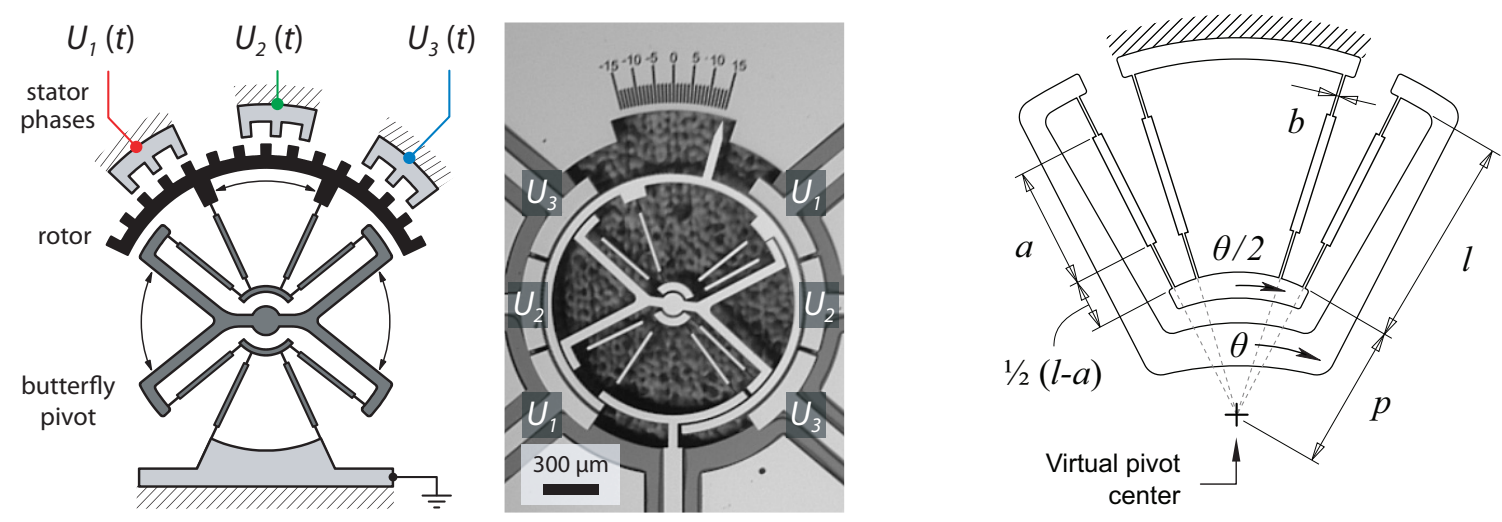

Figure 1: (left) Schematic principle of the 3-phase electrostatic stepper motor (actuated with voltages $U_{1}, U_{2}, U_{3}$ ). The internal "butterfly" structure mechanically connects the rotor to the chassis. (center) Micrograph of the fabricated SOI micromotor rotated clockwise by $+15^{\circ}$. (right) Schematic of a flexural pivot element consisting of four reinforced beams. 

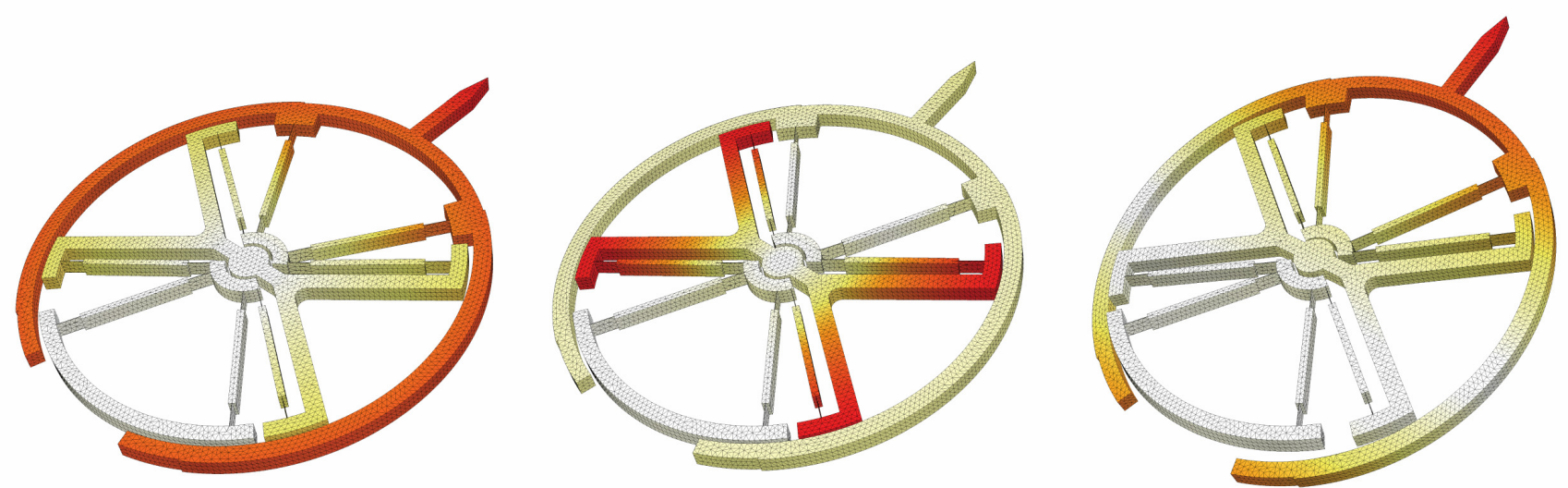

Figure 2: SolidWorks finite element modal analysis of the rotor's mechanical structure. The color levels describe the displacement magnitude relative to the anchor. From left to right, we show the first eigenmodes occurring at $170 \mathrm{~Hz}, 683 \mathrm{~Hz}$ and $2810 \mathrm{~Hz}$, respectively. The first mode corresponds to the in-plane vibration of the rotor ring, while the second mode corresponds to the in-plane vibration of the internal butterfly pivot.

We verify that, in the case of thin flexure beams of length $l$ ( $a=0)$, equation (1) can be rewritten as [1,2]:

$$
\left.K_{\theta}\right|_{a=0}=\frac{4 E I}{l}\left(1+3 \frac{p}{l}+3 \frac{p^{2}}{l^{2}}\right) .
$$

On the other hand, when $p \rightarrow \infty$, one can find the stiffness of a folded-beam flexure with reinforced beams. Defining the tangential displacement $x \equiv p \theta$, we find the stiffness of the folded-beam linear guiding [3]:

$$
K_{x}=\lim _{p \rightarrow \infty} \frac{K_{\theta}}{p^{2}}=\frac{12 E I}{(l-a)\left(a^{2}+a l+l^{2}\right)} .
$$

\section{Finite element analysis}

For the dynamic analysis of the mechanical structure, we have performed FEA simulations with SolidWorks Simulation software (SolidWorks 2009 SP5.0). Because of the crystallographic nature of silicon, the material has orthotropic elasticity. In our simulations, we have therefore used the orthotropic matrix representation of single-crystal silicon, using the parameters recommended by Hopcroft et al. [4]. The results of the modal analysis study are shown in Figure 2. For these simulations, we have considered flexure beams with a width of $1.15 \mu \mathrm{m}$. This dimension, which is within the optical measurement uncertainty, was chosen so as to obtain the best match between the simulated result and the experimental data for the first eigenfrequency. With this parameter, the first mode occurs around $170 \mathrm{~Hz}$ and corresponds to the in-plane vibration of the rotor ring, while the second mode occurs around $683 \mathrm{~Hz}$ and corresponds to the in-plane vibration of the internal butterfly pivot.

\section{MICROFABRICATION RESULTS}

The micromotor was fabricated in a two-mask process using standard SOI micromachining. Figure 3 shows scanning electron microscopy micrographs of a fabricated device. The fabrication process was performed on a (100)-oriented SOI wafer with a device layer of $50 \mu \mathrm{m}$, a buried oxide layer of $2 \mu \mathrm{m}$, and a handling substrate of 380 $\mu \mathrm{m}$. On the device layer, a $100 \mathrm{~nm}$-thick aluminum layer was evaporated, then patterned by ICP (Surface Technology Systems, STS Multiplex ICP Etch). Subsequently, the device layer and the handling wafer were etched by DRIE (Adixen Dielectric and Silicon Etcher, AMS 200 DSE) using the Bosch process. After etching, the buried oxide beneath the rotor was removed using vapor HF (Idonus HF Vapor Phase Etcher, VPE-100). Finally, the silicon chip was mounted on a Printed Circuit Board and wire-bonded.

Note that the fabrication process could be straightforwardly simplified using a single-mask process, as we have demonstrated in an earlier paper [5]. However, such a fabrication process implies the use of honeycomb-like structures. Here, the selection of a two-mask process was motivated by the need for massive structures that can be
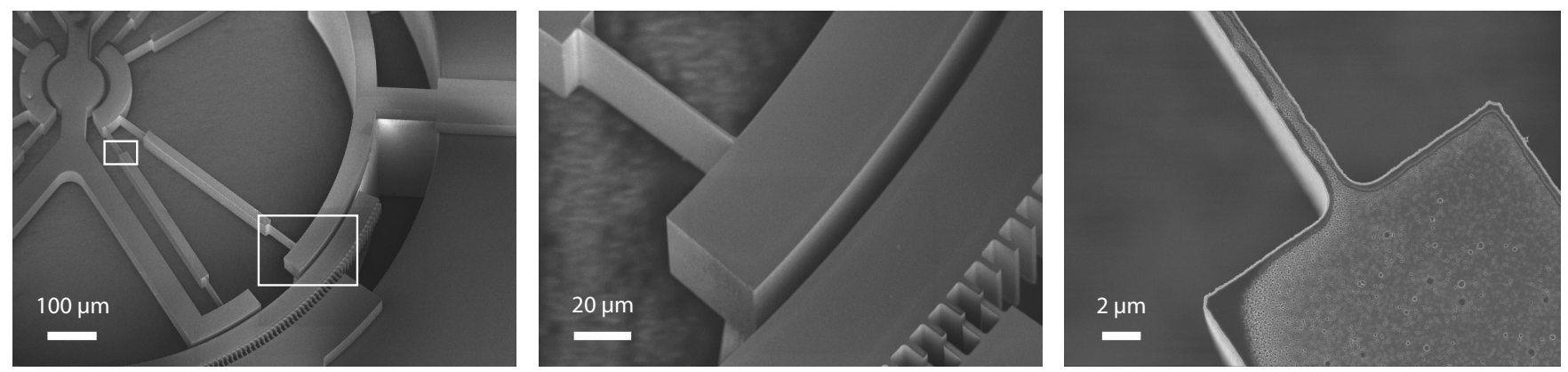

Figure 3: Scanning electron microscopy micrographs of a 3-phase electrostatic stepper micromotor fabricated with SOI technology. The dimensions of the flexure beams were estimated from these observations. 
easily modeled analytically and simulated with FEA software.

\section{EXPERIMENTAL RESULTS \\ Quasi-static and transient responses}

In Figure 4, we show quasi-static measurements performed with a 3-phase sinusoidal driving sequence at $1 \mathrm{~Hz}$. The maximum angular displacement increases quadratically with the applied voltage, as is expected for an electrostatic actuator [1].

Figure 5 is the transient response obtained by releasing the rotor from a small angle to its resting position. The damping coefficient and the first in-plane resonant frequency have been extracted from these data. Figure 6 exhibits a typical motion of the rotor rotated from $0^{\circ}$ to $+9^{\circ}$. An angular speed of $1 \% \mathrm{~ms}$ could be reached with a driving signal of $600 \mathrm{~Hz}$ and $70 \mathrm{~V}$ amplitude. The measurements shown in Figures 5 and 6 were performed with a high-speed camera (Mikrotron CMOS high-speed camera, EoSens MC1363), using the algorithm described in [6].

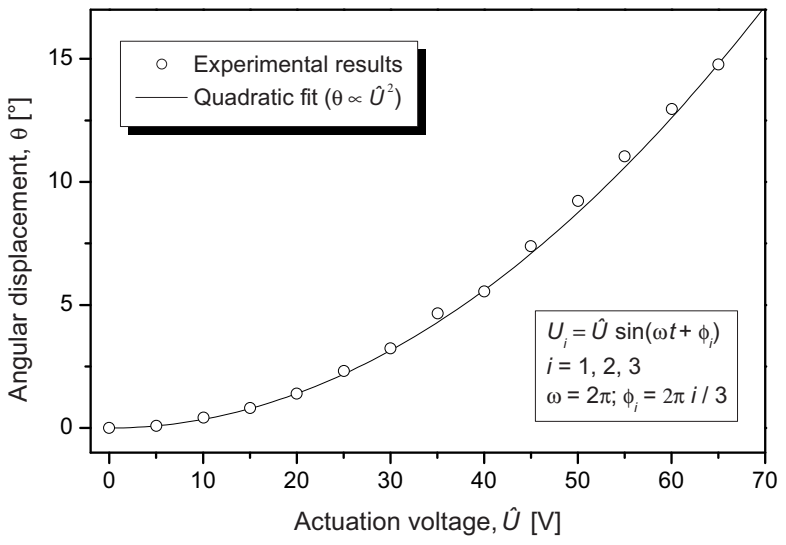

Figure 4: Maximum angular displacement of the stepper motor as a function of the driving voltage (3-phase sinusoidal excitation of $1 \mathrm{~Hz}$ ). Data are for positive displacements only: The displacement range is double.

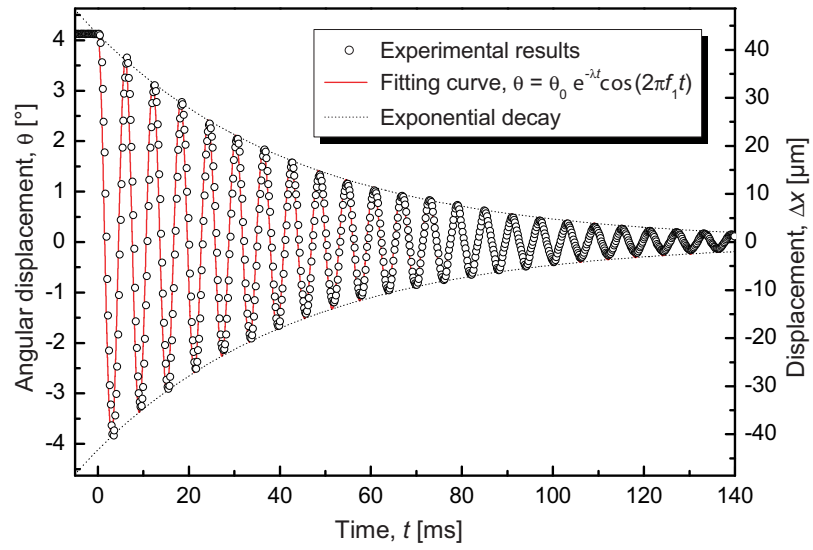

Figure 5: Step response of the rotor. The measurements were performed on the rotor's external ring. The first resonant frequency of $163 \mathrm{~Hz}$ can be extracted from the Fourier transform of these data.

\section{Experimental modal analysis}

We have performed a thorough in-plane and out-ofplane modal analysis of the structure with the Polytec Micro System Analyzer MSA-400. Figure 7 shows the in-plane data recorded by stroboscopic video microscopy under application of a swept excitation voltage on phase 2 . The out-of-plane response (not shown in this abstract) was obtained by Laser-Doppler vibrometry. The results of the FEA simulations and the experimental modal analysis are summarized in Table 1. This table highlights the high conformity of the simulations for the first 5 modes (with a discrepancy lower than $10 \%$ ).

\section{DISCUSSION}

From the in-plane experimental modal analysis, we could retrieve the first two resonant frequencies $f_{1}$ and $f_{2}$ of the motor, which correspond to the eigenfrequencies of the rotor $\left(f_{1}\right)$ and of the "butterfly" internal structure $\left(f_{2}\right)$, as shown in the simulations of Figure 2.

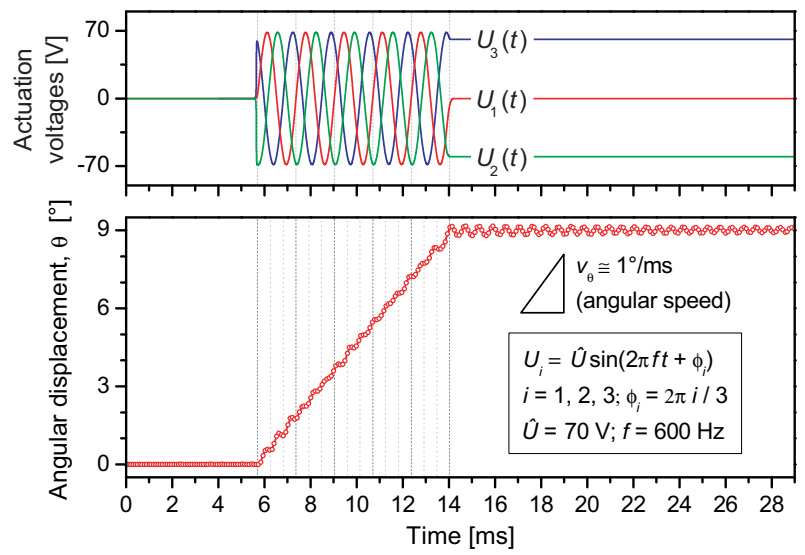

Figure 6: Typical recording of the angular displacement as a function of time. A 3-phase sinusoidal driving signal at $600 \mathrm{~Hz}$ and $70 \mathrm{~V}$ was applied, as illustrated in the upper part of the graph. With these settings, an angular speed of $1 \%$ ms was reached.

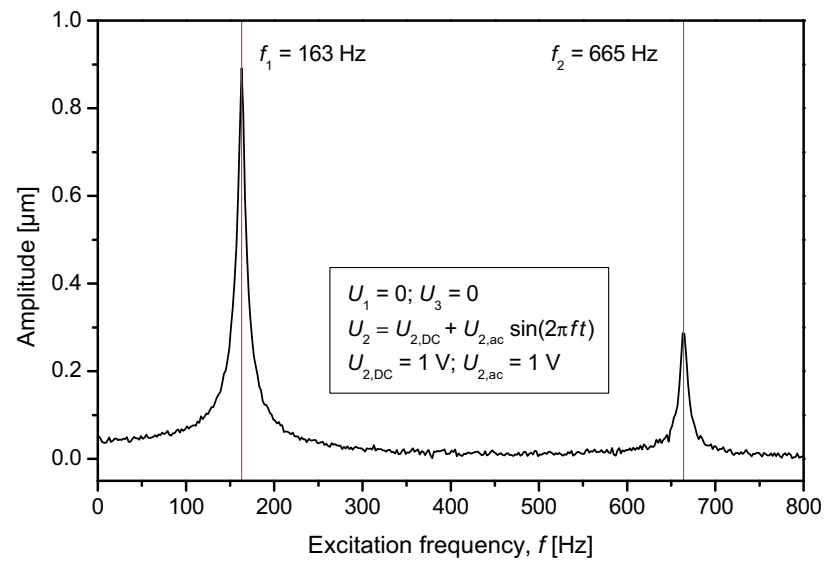

Figure 7: In-plane modal analysis of the micromotor recorded by stroboscopic video microscopy (Polytec MSA400). These results are in line with the transient measurement of Figure 5. 
Table 1: Design parameters and main modal properties of the micromotor. FEA simulations were obtained with SolidWorks Simulation software. Measurements were performed with the Polytec Micro System Analyzer MSA-400.

\begin{tabular}{|c|c|c|c|c|c|}
\hline \multirow{2}{*}{\multicolumn{2}{|c|}{ Main geometric characteristics }} & \multicolumn{4}{|c|}{ Modal analysis } \\
\hline & & Mode & FEA simulations & Experimental results & Remarks \\
\hline \multicolumn{2}{|c|}{ SOI: (100) orientation*; $50 \mu \mathrm{m} / 2 \mu \mathrm{m} / 380 \mu \mathrm{m}$} & 1 & $170 \mathrm{~Hz}$ & $163 \mathrm{~Hz} \pm 1 \mathrm{~Hz}$ & In-plane \\
\hline Rotor diameter & $1.4 \mathrm{~mm}$ & 2 & $683 \mathrm{~Hz}$ & $665 \mathrm{~Hz} \pm 1 \mathrm{~Hz}$ & In-plane \\
\hline Flexure hinge width & $b=1.5 \mu \mathrm{m} \pm 0.4 \mu \mathrm{m} * *$ & 3 & $2810 \mathrm{~Hz}$ & $2616.4 \mathrm{~Hz}$ & Out-of-plane \\
\hline No. of teeth per phase & 80 & 4 & $5350 \mathrm{~Hz}$ & $5018.0 \mathrm{~Hz}$ & Out-of-plane \\
\hline Rotor / stator gap & $2 \mu \mathrm{m} \pm 0.4 \mu \mathrm{m}$ & 5 & $6110 \mathrm{~Hz}$ & $5726.6 \mathrm{~Hz}$ & Out-of-plane \\
\hline
\end{tabular}

* The reader is referred to Table VI in [4] for the elastic modulus values of single-crystal silicon material.

** In the FEA simulations, we have used a beam width of $1.15 \mu \mathrm{m}$.

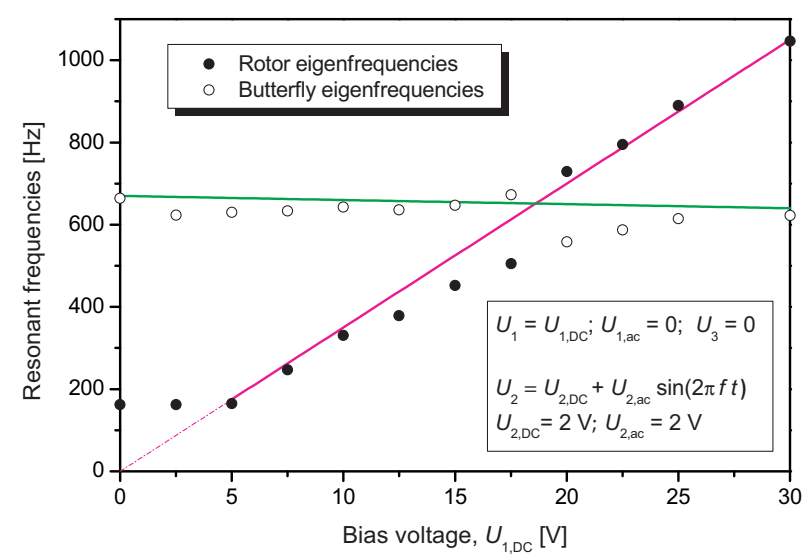

Figure 8: Illustration of the electrostatic stiffening. The rotor eigenfrequency increases when a DC voltage is applied on phase 1 .

In Figure 8, we demonstrate that the first resonant frequency can be tuned by "electrostatic stiffening" [7]. The graph was obtained from data like those shown in Figure 7. When a DC voltage is applied on phase 1 ( $U_{1}=$ const.) while the ac excitation is performed on phase 2 , an electrostatic force tends to keep the rotor's teeth aligned with phase 1 . The rotor is directly affected by the stiffening while the butterfly - which is mechanically connected with flexure beams to the rotor (see Figure 1) - keeps its own degree of freedom, even when the rotor is firmly blocked. As a result, $f_{2}$ remains relatively constant while $f_{1}$ increases almost linearly with $U_{1}$. This linear behavior is consistent with the equation of motion (see calculation of the electrostatic spring stiffness in [7]).

\section{CONCLUSION AND OUTLOOK}

With a displacement range of $+/-15^{\circ}$, our micromotor opens perspectives in reliable and frictionless micrometerscale displacement applications. It is a suitable candidate for a micromanipulator (e.g. bio-MEMS tweezers). Furthermore, the well understood and tunable dynamic response enables accurate micropositioning in microrobotics.

In Lausanne, we are currently working towards the optimization and implementation of several stepper motors for microrobotic applications. On the other side, in Tokyo, a numerical analysis of the complete system consisting of the mechanical structure and the variable-capacitance actuation is performed [8].

\section{ACKNOWLEDGMENTS}

This research was partially supported by the Swiss National Science Foundation through its Ambizione funding program (Grant No. PZ00P2_121827).

The microfabrication was performed by Meena Kulkarni in the cleanroom of the EPFL Center of MicroNanoTechnology (CMi). The authors are thankful to the CMi staff for their technical and scientific support.

\section{REFERENCES}

[1] E. Sarajlic, C. Yamahata, M. Cordero, H. Fujita, "Three-Phase Electrostatic Rotary Stepper Micromotor with a Flexural Pivot Bearing", J. Microelectomech. Syst., vol. 19, pp. 338-349, 2010.

[2] S. Henein, P. Spanoudakis, S. Droz, L.I. Myklebust, E. Onillon, "Flexure Pivot for Aerospace Mechanisms", in Proc. $10^{\text {th }}$ European Space Mechanisms \& Tribology Symposium, San Sebastián, Spain, 2003, pp. 285-288.

[3] S. Henein, Conception des guidages flexibles, PPUR, Lausanne, Switzerland, 2004.

[4] M.A. Hopcroft, W.D. Nix, T.W. Kenny, "What is the Young's Modulus of Silicon?", J. Microelectomech. Syst., vol. 19, pp. 229-238, 2010.

[5] E. Sarajlic, C. Yamahata, M. Cordero, L. Jalabert, T. Iizuka, H. Fujita, "Single Mask 3-Phase Electrostatic Rotary Stepper Micromotor", in Proc. $15^{\text {th }}$ Int. Conf. on Solid-State Sensors, Actuators and Microsystems (Transducers '09), Denver, USA, 2009 pp. 1505-1508.

[6] C. Yamahata, E. Sarajlic, G.J.M. Krijnen, M.A.M. Gijs, "Subnanometer Translation of Microelectromechanical Systems Measured by Discrete Fourier Analysis of CCD Images", J. Microelectomech. Syst., vol. 19, pp. 1273-1275, 2010.

[7] W.C. Tang, M.G. Lim, R.T. Howe, "Electrostatic Comb Drive Levitation and Control Method", $J$. Microelectomech. Syst., vol. 1, pp. 170-178, 1992.

[8] J. Sone, T. Mizuma, M. Masunaga, S. Mochizuki, E. Sarajlic, C. Yamahata, H. Fujita, "Analysis of the Characteristics of a Rotary Stepper Micromotor", IEEJ Trans. Sens. Micromach., vol. 130, pp. 310-316, 2010. 